Original article

Ukr Neurosurg J. 2021;27(2):25-33

doi: $10.25305 /$ unj. 226745

\title{
Cranial nerve function after cerebellopontine angle meningiomas removal
}

Andrii H. Sirko ${ }^{1,2}$, Rostislav R. Malyi ${ }^{2}$

${ }^{1}$ Neurosurgery Department No.2, Mechnikov Dnipropetrovsk Regional Clinical Hospital, Dnipro, Ukraine ${ }^{2}$ Neurology and Neurosurgery Department, Postgraduate Education Faculty, Dnipro State Medical University, Dnipro, Ukraine

Received: 14 March 2021

Accepted: 25 May 2021

Address for correspondence: Rostislav R. Malyi, Cerebral Neurosurgery Department No. 2, Mechnikov Dnipropetrovsk Regional Clinical Hospital, 14 Soborna Square, Dnipro, 49005, Ukraine, e-mail: rostikmalyy@gmail.com
Objective. To analyze cranial nerves ( $\mathrm{CN}$ ) dysfunction incidence in cerebellopontine angle (CPA) meningiomas removal depending on topographic and anatomical tumor location type and define the ways to reduce $\mathrm{CN}$ dysfunction incidence and severity.

Materials and methods. The study included 30 CPA meningioma patients operated on in the clinic over a 10-year period (from 2010 to 2020 inclusive). Tumor characteristics, the degree of extent (including matrix location, particularly in relation to the internal auditory canal (IAC), jugular foramen (JF), and Meckel's cave), and supratentorial extension were assessed. The tumors were divided into 5 groups according to the classification of Nakamura et al. Particular attention was paid to the presence and severity of pre- and postoperative cranial nerves dysfunction.

Results. Different groups of CPA meningiomas in terms of the presence of a new CN neurological deficit were compared in the study. CN VII dysfunction was more frequently detected in group 2 - meningiomas extending to IAC, which was $54.5 \%$ of all observed tumors in this group vs. premeatal tumors, where new CN VII deficit was $20 \%$, in group 1 . At the same time in groups 3 , 4 , and 5 there was no new CN VII deficit.

Conclusions. The most frequent symptoms in patients of all groups were dizziness, headache and unsteady gait. Of all the symptoms, only unsteady gait and hearing impairment were more common in patients in group 2 , however the differences were statistically insignificant $(p=0.135$ and $p=0.268$, respectively). Trigeminal nerve and auditory nerve were most commonly affected. Auditory nerve lesions were more often detected in patients of group 2 than in patients of other groups, however, the differences were statistically insignificant $(p=0.268)$. In general, there were no statistically significant differences between the groups in terms of the incidence of various symptoms and the incidence of CPA cranial nerve damage.

Keywords: cerebellopontine angle; auditory nerve; facial nerve; meningioma

\section{Introduction}

Posterior cranial fossa meningiomas account for $\sim 10 \%$ of all intracranial meningiomas [1] Cerebellopontine angle (CPA) meningiomas are the second most common CPA tumors after vestibular schwannomas, accounting for $6 \%$ to $15 \%$ of all CPA tumors and $40 \%$ of all infratentorial meningiomas. $[2,3,4]$. CPA meningiomas are tumors growing from the dura mater (DM) of the posterior surface of the pyramid laterally from the trigeminal nerve [15]. Cranial nerves (CN) function preservation is an important challenge in the surgery of this pathology. Based on the literature data, the following factors affect the cranial nerves function: topographic and anatomical tumor location, its relationship with the surrounding neurovascular structures and preoperative functional state of the $\mathrm{CN}$. When classifying such tumors, special attention is paid to tumor location relative to internal auditory canal. Therefore, there are pre- and postmeatal CPA meningiomas $[9,15]$. Tumor group detection is important in determining the risk of cranial nerve dysfunction development.
Objective: To analyze $\mathrm{CN}$ dysfunction incidence in CPA meningiomas removal depending on topographic and anatomical tumor location type and define the ways to reduce $\mathrm{CN}$ dysfunction incidence and severity.

\section{Materials and methods \\ Participants}

The single-center retrospective study included 30 CPA meningioma patients operated on in the clinic over a 10-year period (from 2010 to 2020 inclusive). All patients were treated in the Neurosurgery Department No. 2, Mechnikov Dnipropetrovsk Regional Clinical Hospital, Dnipro, Ukraine. All patients were operated on by the professor Andrii $\mathrm{H}$. Sirko. The data for the study was selected based on current CPA meningiomas definition and classification of Nakamura et al. (Table 1). Additionally, English-language web sources related to CPA meningiomas diagnosis and treatment from 2000 to 2020 inclusive were analyzed.

Data on the patients, their demography, complaints, symptoms, pre-, intra- and postoperative tumor 
Table 1. Nakamura et al. classification [9]

\begin{tabular}{|l|l|}
\hline Group 1 & Matrix anterior to internal auditory canal \\
\hline Group 2 & Matrix invades internal auditory canal \\
\hline Group 3 & Matrix superior to internal auditory canal \\
\hline Group 4 & Matrix inferior to internal auditory canal \\
\hline Group 5 & Matrix posterior to internal auditory canal \\
\hline
\end{tabular}

characteristics, surgical intervention parameters, postoperative patient condition, complications and longterm consequences of the disease were obtained from the surgeon's database and medical records.

24 women $(80 \%)$ and 6 men (20\%) were operated on. The operated patients' age ranged from 26 to 81 (average, 53.9 \pm 2.3 ). After histopathological examination, all removed tumors were assigned the WHO grade I. There was no statistical difference in tumor lateralization ( $46.6 \%$ of meningiomas were right-sided and $53.4 \%$ left-sided).

All patients received informed and voluntary written consent to participate in the study.

The study was approved by the Commitee on Ethics and Bioethics of Dnipro State Medical University (Meeting Minutes No.1 of February 10, 2020). The work is a fragment of research work (state registration number 0119U000110).

\section{Inclusion criteria}

The study included patients who meet the definition of CPA meningiomas given in the current literature. CPA meningiomas are tumors growing from the dura mater (DM) of the posterior surface of the pyramid laterally from the trigeminal nerve [15].

\section{Characteristics of groups}

Based on neuroimaging studies and intraoperative findings, all patients operated by us were classified as follows as according to classification of Nakamura et al.: group 1, 10 (33.3\%) patients; group 2, 11 (36.6\%) patients; group 3, 7 (23.3\%) patients. Groups 4 and 5 had $1(3.3 \%)$ patient each.

The tumors were primarily divided into groups based on intraoperative findings and neuro-radiological characteristics. Tumor classification according to Nakamura et al. was based on the location of the more vascularized site of tumor attachment (meningioma matrix). Group 1 patients matrix was located on the shell of the posterior surface of the temporal bone pyramid anterior to the IAC, in most cases it passed to the tentorium with the superior petrosal sinus involvement and into the Meckel's cave and also supratentorially (Fig. 1). In most cases, CN VII and VIII were shifted posteriorly, CN V anteriorly, inferiorly and, in some patients, laterally. Group 2 meningiomas directly invaded the IAC or overgrew it from all sides. CN VII and VIII were most often shifted caudally or passed through the tumor (Fig. 2). Group 3 tumors matrix was located on the membrane of the temporal bone pyramid superiorly to the IAC, often passing to the tentorium; the tumor shifted the acoustic and facial nerves inferiorly (Fig. 3). Group 4 tumor was located inframeatally and grew into the jugular foramen (Fig. 4). Group 5 tumor matrix was located posterior to internal auditory canal (Fig. 5)

\section{Study design}

Standard preoperative patients examination included: cliniconeurological and otoneurological examination, brain MRI using Toshiba Excelart Vantage 1.5T XGV (T1, T2, Flair, DWI, AX 3D SSFP IAC - to visualize the cranial nerves; IV contrast (Tomovist 0.4 $\mathrm{ml} / \mathrm{kg}$ ) brain MRI; special program brain CT angiography (Toshiba Aquilion 64-64, contrast agent: Tomohexol 300, 40-60 ml).

Tumor characteristics, spread, including matrix location (particularly relative to the internal auditory canal (IAC), jugular foramen (JO), and Meckel's cave), supratentorial spread, secondary disorders (hydrocephalus), and the need for preoperative preparation (CSF shunt installation, preoperative feed vessels embolization) were assessed. Total removal was confirmed by the absence of the tumor residue on postoperative follow-up CT/MRI (with IV contrast) and was also assessed intraoperatively according to the Simpson grading system.

The tumors were divided into the following groups based on their size: 11-20 mm=medium,

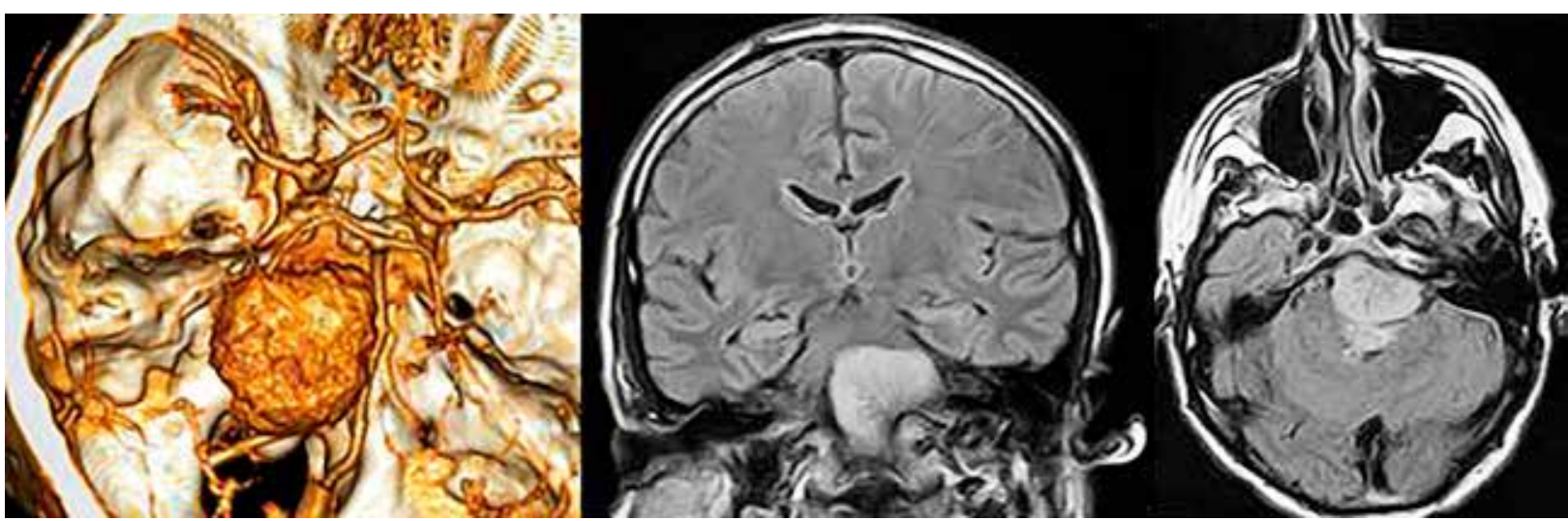

Figure 1. Group 1 patient CT angiography and MRI. Tumor matrix is located at the transition from the posterior face of the pyramid to the clivus (premeatally). 


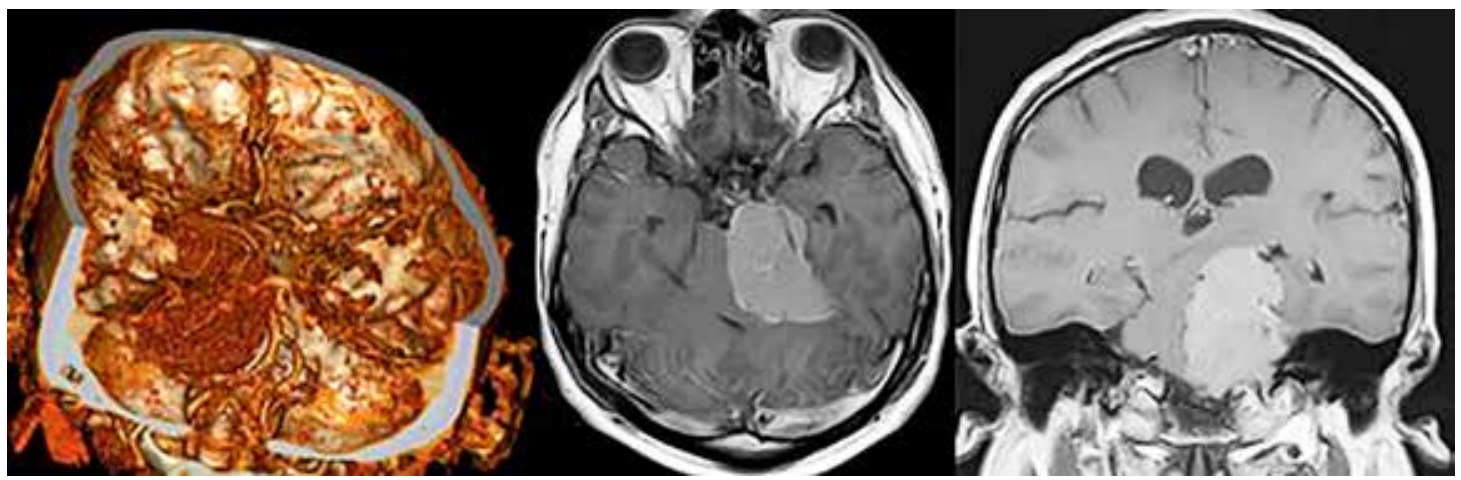

Figure 2. Group 2 patient CT angiography and MRI. Tumor matrix on the dura mater is anterior and superior to the internal auditory canal. The main part of the matrix is in the area of superior petrosal sinus on the membrane at the transition from the tentorium to the posterior surface of the temporal bone pyramid. The facial nerve was shifted by the tumor caudally and inferiorly, the trigeminal nerve anteriorly, the trochlear nerve anteriorly and superiorly.

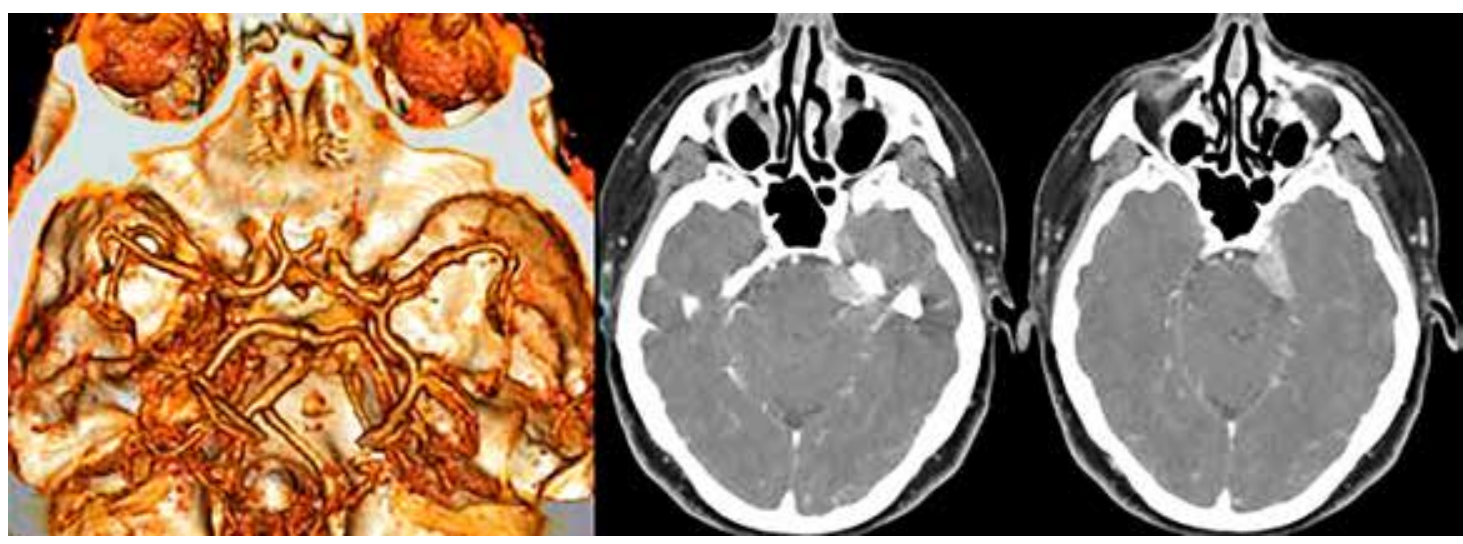

Figure 3. Group 3 patient CT angiography. Tumor matrix is located the transition from the posterior face of the pyramid to the tentorium (suprameatally) with expansion into the Meckel's cave and middle cranial fossa (MCF).
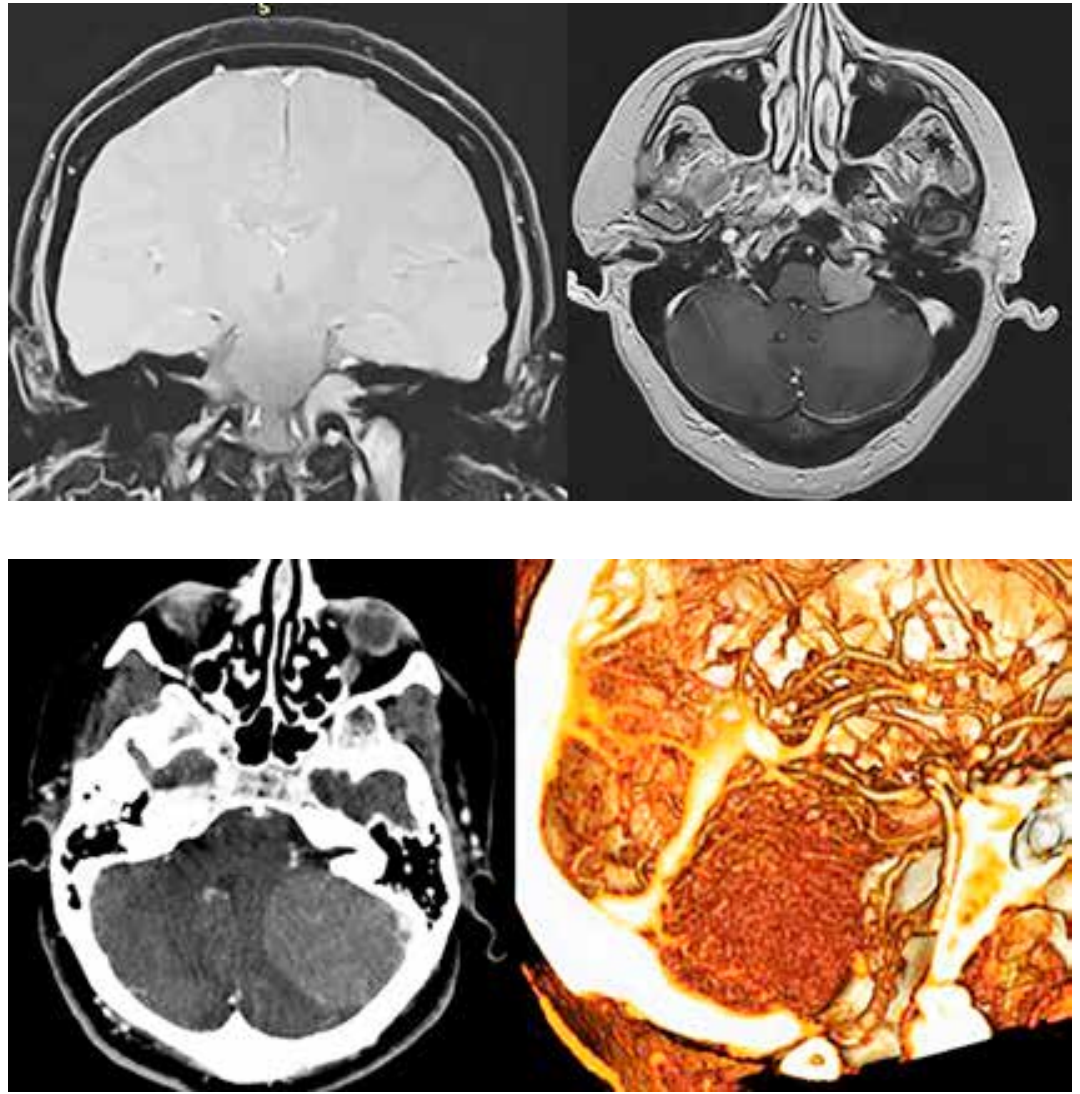

Figure 4. Group 4 patient MRI. Tumor matrix is located anteriorly and superiorly to the jugular foramen (inframeatally). The tumor partially overgrew the caudal $\mathrm{CN}$ group and the facial nerve.
Figure 5. Group 5 patient CT angiography. Tumor matrix was located on the transverse sinus walls, superior petrosal sinus, tentorium, and posterior face of the temporal bone pyramid posteriorly to the internal auditory canal. 
21-30 mm = moderately large, 31-40 mm=large, $>40 \mathrm{~mm}=$ giant meningiomas. For giant tumor sizes, tumor classification was based on intraoperative matrix location findings.

CPA meningiomas were removed in lateral prone position using retrosigmoid approach with the following equipment: microscopes (OPMI VARIO 700 and S88, Carl Zeiss), electric drills (Aesculap, Stryker), ultrasound aspiration dissector (Söring), high-frequency coagulation device (Söring, Codman) using ISOCOOL (Codman) bipolar coagulation forceps, and NIM 2.0/NIM 3.0 intraoperative nerve monitoring system (Medtronic). A four-channel stimulation mode for facial nerve monitoring with a monopolar electrode through the perineural tumor tissue and/or direct facial nerve stimulation were applied. A $1.0 \mathrm{~mA}$ current was initially used for direct stimulation, with gradual decrease of current strength to $0.05 \mathrm{~mA}$ (event recording threshold, $100 \mu \mathrm{V}$ ). In case of initial deficiency or in elderly patients: initial stimulation current was 1.2 to $1.5 \mathrm{~mA}$; event recording threshold, 80-90 $\mu \mathrm{V}$. The monitoring (a free-running EMG) was also performed based on spontaneous nerves activity.

Particular attention was paid to the presence and severity of pre- and postoperative cranial nerves dysfunction.

\section{Statistical methods}

Statistical analysis was carried out in addition to the "Package Analysis" Microsoft Excel. The significance of difference between groups was assessed using the $x 2$ test. Values were considered statistically significant at $\mathrm{p}<0.05$

\section{Results}

Moderately large CPA meningiomas were diagnosed in $8(26.6 \%)$ cases, large in $10(33.4 \%)$ cases, giant in 12 (40\%) cases. Thus, large and giant meningiomas, which accounted for $73.4 \%$ of all meningiomas, prevailed in the study. Average mediolateral tumor size was $30 \pm 2$ $\mathrm{mm}$, anteroposterior size $37.2 \pm 2.2 \mathrm{~mm}$, rostrocaudal size $30.4 \pm 1.9 \mathrm{~mm}$.

$7(23.3 \%)$ patients had supratentorial tumor spread. In $10(33.3 \%)$ patients, the tumor spread into the Meckel's cave. 5 (16.6\%) patients had preoperative hydrocephalus.

Average preoperative symptoms duration was $9.25 \pm 2.27$ months (1.5 to 60 months).

The most frequent symptoms were: dizziness, in $19(63.3 \%)$ patients; headache, in 15 patients (50\%); unsteady gait, in 15 (50\%) patients. (Fig 6). In cases of clinical manifestations of trigeminal nerve damage: $7(23.3 \%)$ patients had numbness or facial hypesthesia, $4(13,3 \%)$ patients had trigeminal neuralgia. In those patients, neuroimaging revealed the meningioma's invasion into the Meckel's cave and MCF. In 4 patients (13.3\%) with clinical manifestations of hemiparesis/ hemihypesthesia, the MRI showed peritumoral edema and stem structures shift; the patients also needed pre- or intraoperative hydrocephalus management (cerebrospinal fluid (CFS) or ventriculocisternal shunt installation according to Torkildsen). 5 (15\%) patients had preoperative CN VII deficiency. 4 (13.3\%) patients, had House-Brackmann grade II CN dysfunction and one patient had grade $V$ dysfunction. 7 (23.3\%) patients had preoperative hearing impairment. Clinical significance 7 and 8 (hearing impairment and palsy) corresponded to the radiological results (tumor invasion into the IAC or shift/close contact with the CN). 2 (6.6\%) patients had preoperative bulbar palsy. Diplopia was identified in 3 $(10 \%)$ cases.

There were no statistically significant differences between the groups in the frequency of occurrence of different symptoms. (Table 2). However, the frequency of unsteady gait and hearing impairment in the groups is less homogeneous. ( $p=0,135$ and $p=0,268$ respectively). These symptoms were more common in patients in group 2 than in patients in other groups.

The trigeminal and auditory nerves were most commonly affected (Fig. 7). This is most likely associated with the location of the matrix in the channels of these nerves, as well as with the more frequent effect of tumor tissue on these nerves.

There were no statistically significant differences between the groups in the frequency of tumor lesions of cranial nerves. (Table 3). However, the frequency

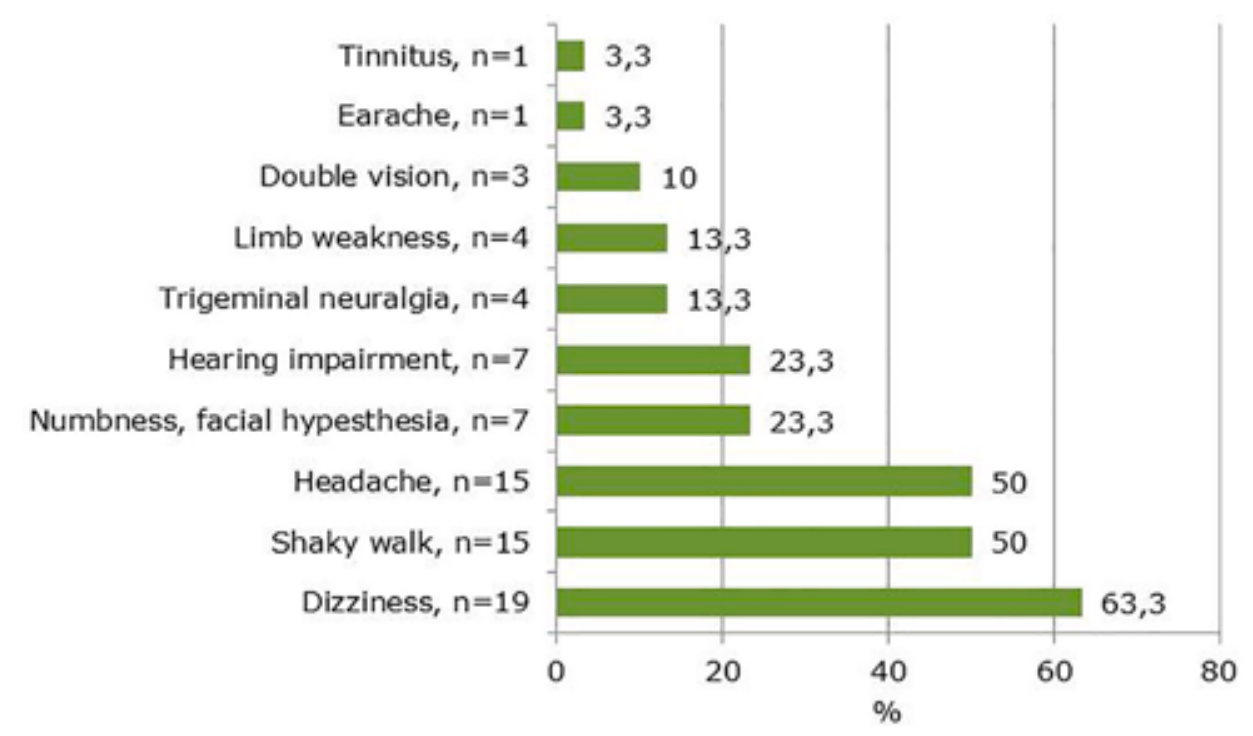

Fig 6. Distribution of symptoms by frequency of occurrence in cerebellopontine meningiomas before surgery 
Table 2. Incidence of various preoperative symptoms in CPA meningioma patients

\begin{tabular}{|c|c|c|c|c|c|c|}
\hline \multirow{2}{*}{ Symptoms } & \multicolumn{5}{|c|}{ Number of cases in groups } & \multirow[b]{2}{*}{$\mathbf{p}$} \\
\hline & $\begin{array}{c}1 \\
n=10\end{array}$ & $\stackrel{2}{n=11}$ & $\stackrel{3}{n=7}$ & $\begin{array}{c}4 \\
n=1\end{array}$ & $\begin{array}{c}5 \\
n=1\end{array}$ & \\
\hline Dizziness & 5 & 8 & 4 & 1 & 1 & 0,638 \\
\hline Unsteady gait & 5 & 8 & 2 & 0 & 0 & 0,135 \\
\hline Headache & 5 & 6 & 3 & 0 & 1 & 0,972 \\
\hline Numbness, facial hypesthesia & 2 & 3 & 2 & 0 & 0 & 0,847 \\
\hline Trigeminal neuralgia & 1 & 1 & 2 & 0 & 0 & 0,579 \\
\hline Limb weakness & 2 & 2 & 0 & 0 & 0 & 0,657 \\
\hline Hearing impairment & 1 & 5 & 1 & 0 & 0 & 0,268 \\
\hline Double vision & 1 & 2 & 0 & 0 & 0 & 0,611 \\
\hline Earache & 1 & 0 & 0 & 0 & 0 & 0,875 \\
\hline Tinnitus & 0 & 1 & 0 & 0 & 0 & 0,695 \\
\hline
\end{tabular}

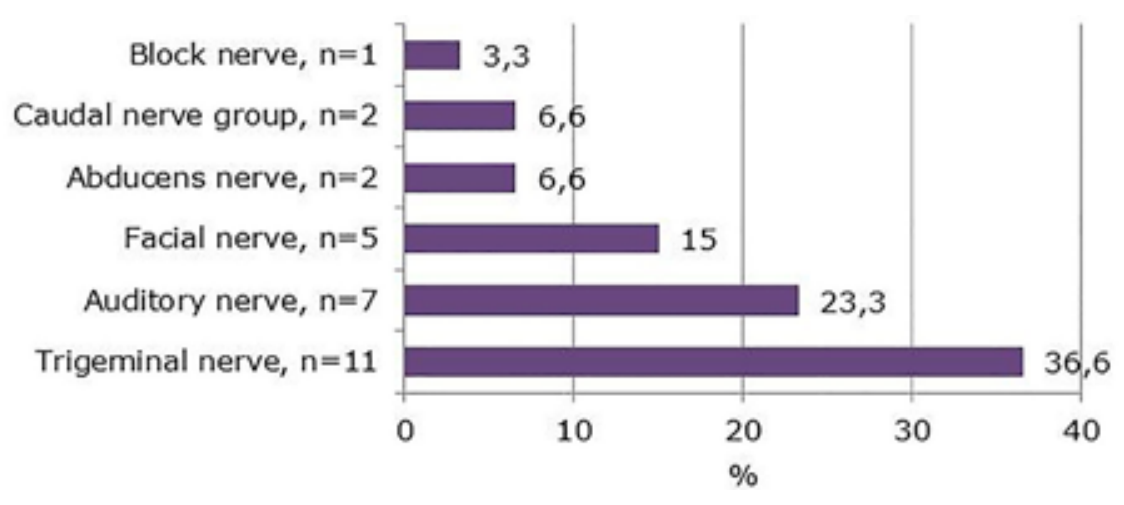

Fig 7. Distribution of cranial nerves according to the frequency of lesions in CPA meningioma before surgery

Table 3. Incidence of cranial nerve lesions in CPA meningioma patients

\begin{tabular}{|c|c|c|c|c|c|c|}
\hline \multirow[b]{2}{*}{ Nerves } & \multicolumn{5}{|c|}{ Number of cases in groups } & \multirow[b]{2}{*}{$\mathbf{p}$} \\
\hline & $\begin{array}{c}1 \\
n=10\end{array}$ & $\begin{array}{c}2 \\
n=11\end{array}$ & $\stackrel{3}{=} 7$ & $\begin{array}{c}4 \\
n=1\end{array}$ & $\begin{array}{c}5 \\
n=1\end{array}$ & \\
\hline Trigeminal nerve & 3 & 4 & 4 & 0 & 0 & 0,418 \\
\hline Auditory nerve & 1 & 5 & 1 & 0 & 0 & 0,268 \\
\hline Facial nerve & 2 & 2 & 1 & 0 & 0 & 0,930 \\
\hline Abducens nerve & 1 & 1 & 0 & 0 & 0 & 0,862 \\
\hline Caudal nerve group & 1 & 0 & 0 & 1 & 0 & 0,062 \\
\hline Block nerve & 1 & 0 & 0 & 0 & 0 & 0,884 \\
\hline
\end{tabular}

of auditory nerve and caudal nerve group damage in the groups is less homogeneous. $(p=0,268$ and $p=0,062$ respectively). Patients in group 2 were more likely to have auditory nerve damage than patients in other groups. According to caudal nerve group, such a conclusion cannot be drawn, there is only one patient in group 4.

In 27 of $30(90 \%)$ cases, total resection of Simpson Grade II meningioma was performed (total resection of the tumor's nodular part with matrix coagulation on the membrane of the posterior face of the pyramid, clivus, excision or coagulation of the tentorium or venous sinus walls). Subtotal CPA meningiomas resection was performed in three cases. In one case of subtotal resection (Simpson IV), meningioma removal was abandoned due to development of drug-resistant bradycardia; in other cases, the tumor strongly adhered to the brain stem and cranial nerves. In 29 of 30 cases, the tumor was removed using a retrosigmoid approach. In one case, an extended retrosigmoid approach with craniovertebral junction decompression was applied (resection of the posterior semicircle of the foramen magnum and the $\mathrm{C} 1$ vertebral 
arch, which was performed due to the large tumor size to prevent postoperative complications (brainstem structures compression, occlusive hydrocephalus due to cerebellar edema) (Fig. 8).

In all cases, the duraplasty was performed using a periosteal pedicle graft. $6(23.3 \%)$ patients had occlusive hydrocephalus. To eliminate and prevent the hydrocephalus growth, 2 patients underwent intraoperative ventriculocisternal shunting according to Torkildsen, while 4 patients had the CFS installed (configured at medium pressure) as the first stage (Fig. 9). For one patient without hydrocephalus, considering the large tumor size, brainstem structures shift, IV ventricle compression and shift, and the presence of peritumoral edema, the CFS (configured at medium pressure) was also installed at the first stage.

In the early postoperative period, two (6.6\%) patients developed occlusive hydrocephalus associated with postoperative cerebellar edema; to correct the hydrocephalus, the CFS (configured at medium pressure) was installed in an urgent manner. To remove the
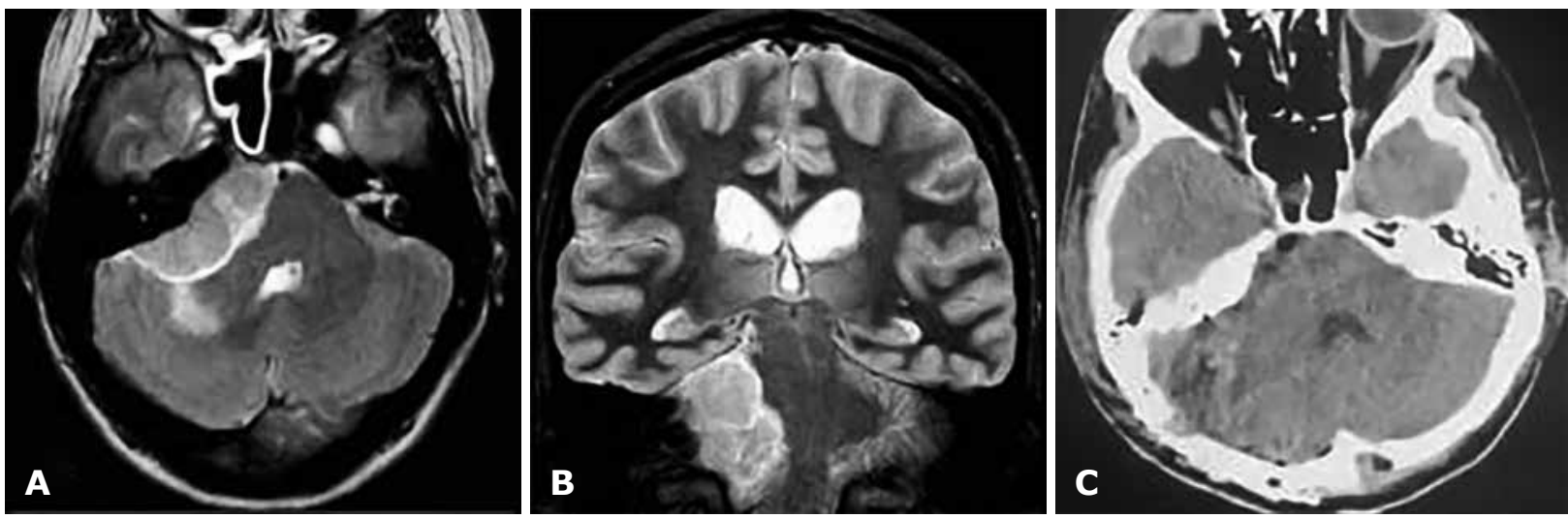

Figure 8. A, B - CPA meningioma MRI in a group 2 patient, who was subjected to extended retrosigmoid approach with the craniovertebral junction decompression (resection of the posterior semicircle of the foramen magnum and the $\mathrm{C} 1$ vertebral arch). $\mathbf{C}$ - Postoperative $\mathrm{CT}$ in the same patient.
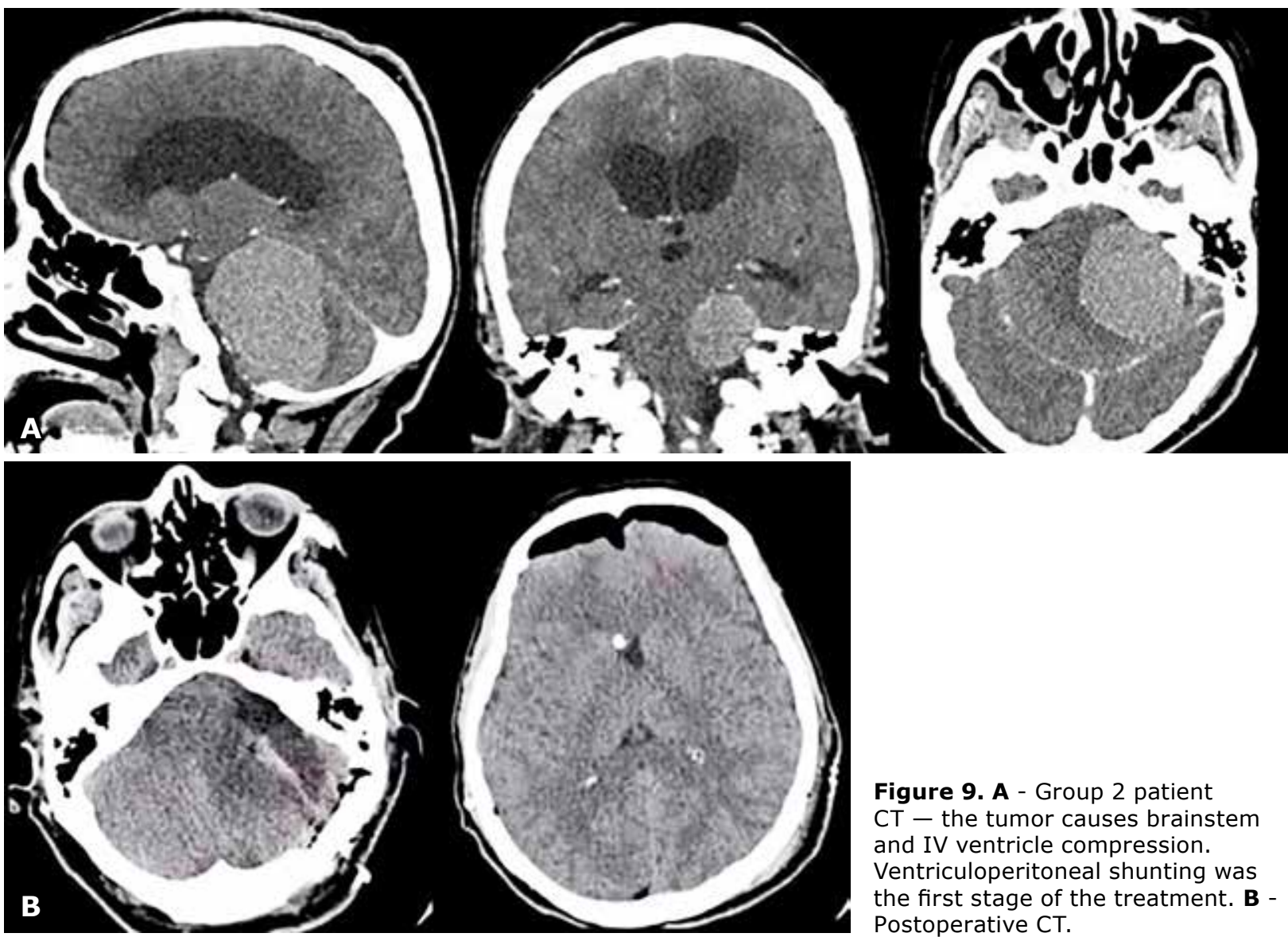

Figure 9. A - Group 2 patient CT - the tumor causes brainstem and IV ventricle compression. Ventriculoperitoneal shunting was the first stage of the treatment. B Postoperative CT. 
supratentorial part of the tumor, tentoriotomy was performed in $3(10 \%)$ cases. In only one case of subtotal tumor removal, the patient underwent linear accelerator radiation therapy.

Most (25 (83.3\%)) patients had no preoperative facial nerve deficit. 7 (23.3\%) patients had hypesthesia in the CN V innervation zone. $4(13,3 \%)$ patients had trigeminal neuralgia. 7 (23.3\%) patients had hearing impairment. $2(6.6 \%)$ patients had bulbar palsy.

All patients underwent neurophysiological monitoring during tumor removal. The anatomical integrity of the CNs was preserved in all cases.

Of 10 operated group 1 (Fig. 10) patients, 2 (20\%) patients had preoperative CN VII dysfunction (House-Brackmann grade II). 2 of 10 (20\%) patients had postoperative dysfunction: One patient had HouseBrackmann grade II dysfunction and the other one had House-Brackmann grade III dysfunction. In one case, the deficit completely regressed after 14 days. 2 (20\%) patients had hypesthesia in the innervation zones of the trigeminal nerve branches I and II. In one of those patients, the deficit regressed after surgery. In one patient, trigeminal neuralgia also completely regressed postoperatively. 1 patient developed a new neurological deficit manifested in hypesthesia in the innervation zone of CN V, branches II and III. Three (30\%) patients had unstable postoperative abducens nerve deficit. One patient had a pronounced postoperative bulbar palsy, due to which the patient underwent tracheotomy. 1 patient had hypoacusis, which did not change postoperatively.

Of 11 operated group 2 (Fig. 11) patients, 2 (18.1\%) patients had preoperative CN VII dysfunction (House-Brackmann grade II and V). 6 of 11 (54.5\%) patients had facial nerve dysfunction. 2 patients had House-Brackmann grade III, 2 patients had HouseBrackmann grade IV, one patient had House-Brackmann grade $V$, and one patient had House-Brackmann grade VI. $2(18.1 \%)$ patients had hypesthesia in the innervation zone of the trigeminal nerve branches. In all patients, the deficit regressed postoperatively. One patient also had complete postoperative regression of clinical manifestations of trigeminal neuralgia, but the same patient had facial hypesthesia after surgery. 4 $(36.3 \%)$ patients had hypoacusis and one $(9 \%)$ patient had complete hearing loss on the affected side. All patients had the same hearing before and after surgery. 5 patients had a CN VI deficit of varying severity, which in most cases regressed.

In 7 operated group 3 patients, 1 patient (14.2\%) had preoperative CN VII dysfunction (House-Brackmann grade II); postoperatively, the deficit had the same grade. $2(28.5 \%)$ patients had hypesthesia in the innervation zones of the trigeminal nerve branches and clinical manifestations of trigeminal nerve neuralgia. Postoperatively, neuralgia regressed in both cases; however, one of the two patients had facial hypesthesia after surgery. 1 (14.2\%) patient had hypoacusis and the same hearing level after surgery.

One group 4 patient had a slightly pronounced postoperative bulbar palsy, which was associated with tumor attachment in the jugular foramen area.

No new $\mathrm{CN}$ deficit was found in the group 5 patient.

In total, a new facial nerve neurological deficit was detected in $8(26.6 \%)$ of 30 patients. Of those, one patient had House-Brackmann grade II, 3 patients had House-Brackmann grade III, 2 patients had HouseBrackmann grade IV, 4 patients had House-Brackmann grade $\mathrm{V}$, and 1 patient had House-Brackmann grade VI. 2 $(6.6 \%)$ of 30 patients had a new trigeminal nerve deficit. All 5 patients with this type of preoperative dysfunction had complete regression of pain syndrome associated with secondary trigeminal neuralgia. $8(26.6 \%)$ patients had postoperative CN VI deficit.

\section{Discussion}

Based on earlier studies, the most common symptoms of CPA meningiomas include unsteady gait, dizziness, hearing impairment, and headache [5], which is consistent with our findings.

According to the literature, the retrosigmoid approach, which was used in all cases of our study, is the most common approach for this particular pathology [5]. However, some sources describe the advantage of the anterior translabyrinthine approach in premeatal tumors [7]. When removing giant tumors, an extended retrosigmoid approach with the craniovertebral junction decompression is used (resection of the posterior semicircle of the foramen magnum and the $\mathrm{C} 1$ vertebral

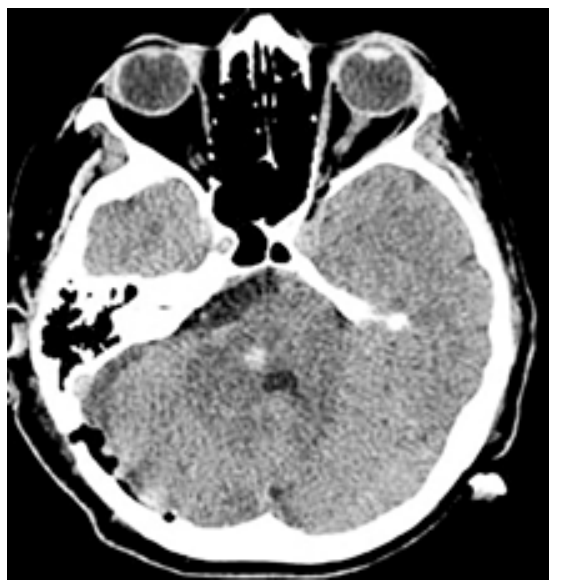

Figure 10. Group 1 patient postoperative CT.

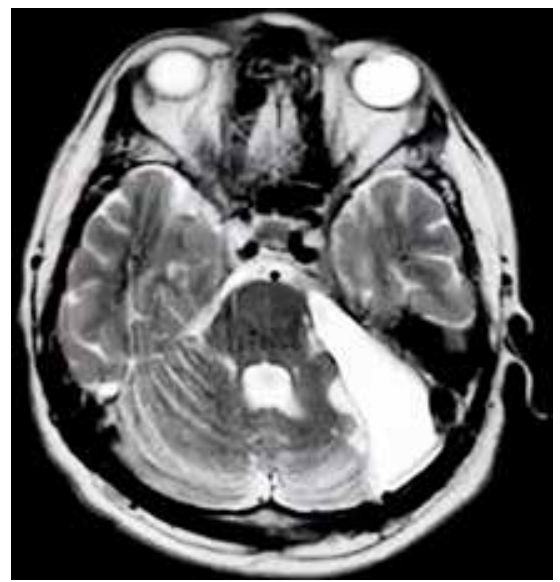

Figure 11. Follow-up MRI in a group 2 patient at 6 months after surgery. 
arch [8]), which provides a clear view for safe tumor removal and reduces the risk of brainstem structures compression. In our study, such approach was applied in one case, where the tumor belonged to group 2 and was over $5 \mathrm{~cm}$ in diameter (see Fig. 8).

According to various sources, the percentage of postoperative facial nerve dysfunction ranges from $6 \%$ to $29 \%[3,4]$. In our study, it was $26.6 \%$. Based on the literature data, postoperative facial and auditory nerves deficits are most often associated with tumor invasion into the IAC; such tumors typically belong to group 2 according to the classification of Nakamura et al. [9].

The literature describes various methods of $\mathrm{CN}$ function preservation, which allows to reduce the incidence of complications. In case of tumor invasion into the IAC, it is decompressed by cutting its walls with a drill (the drilling) to ensure CN VII and CN VIII decompression and more controlled removal of the intrameatal part of the tumor [4]. In case of coarse tumor growth around nerve stems, the method of keeping the residual part of the tumor with subsequent stereotactic radiosurgery is applied $[5,6]$. Postoperative $\mathrm{CN}$ dysfunction is associated with a large tumors size, tumor tissue and matrix invasion into $\mathrm{CN}$ exits from the PCF cavity (Meckel's cave, internal auditory canal, Dorello's canal, jugular foramen), and direct contact between tumor tissue and the CN. At the same time, preservation of anatomical integrity of the nerves does not exclude their postoperative dysfunction.

Different groups of CPA meningiomas in terms of the presence of a new CN neurological deficit were compared in the study. CN VII dysfunction was more frequently detected in group 2 - meningiomas extending to IAC, which was $54.5 \%$ of all observed tumors in this group vs. premeatal tumors, where new CN VII deficit was $20 \%$, in group 1 . Groups 3, 4, and 5 had no new CN VII deficit. The obtained data is consistent with previous studies $[3,5,7,9]$.

Group 4 (inframeatal) patients may have neurological deficit associated with caudal nerves damage more frequently due to tumor invasion into the jugular foramen. Different authors suggest less aggressive removal of this part of the tumor, followed by radiosurgery, since bulbar disorders significantly affect the patients' quality of life [4].

Neurophysiological monitoring is an important tool for preserving $\mathrm{CN}$ function and preventing neurological complications. Currently, the methods such as somatosensory evoked potentials, CN function, motor evoked potentials, and brainstem auditory evoked potentials monitoring, and electroencephalography are used. Neurophysiological monitoring increases the degree of radical resection and reduces the risk of postoperative complications $[4,10,11]$.

Based on the data obtained, CPA meningiomas removal is associated with a large incidence of abducens nerve lesions $-8(26.6 \%)$ in our study. This is particularly significant for large and giant tumors removal. According to the literature, abducens nerve neurophysiological monitoring is of great value for determining the nerve location relative to the tumor but does not greatly affect the preservation of nerve function $[12,13]$.
Brainstem auditory evoked potentials are an important tool for maintaining the vestibulocochlear nerve function. All patients shall undergo audiometry for pre- and postoperative assessment of the auditory analyzer condition [14].

\section{Conclusion}

The most frequent symptoms were dizziness, headache and unsteady gait.

Of all the symptoms, only unsteady gait and hearing impairment were more common in patients in group 2 , but the differences were statistically insignificant ( $p=0.135$ and $p=0.268$, respectively).

Trigeminal nerve and auditory nerve were most commonly affected.

Auditory nerve lesions were more often detected in patients of group 2 than in patients of other groups, however, the differences were statistically insignificant ( $p=0.268)$.

In general, there were no statistically significant differences between the groups in terms of the incidence of various symptoms and the incidence of cranial nerve damage.

\section{Disclosure}

Conflict of interest

The authors declare no conflict of interest.

Ethical approval

All procedures performed in studies involving human participants were in accordance with the ethical standards of the institutional and national research committee and with the 1964 Helsinki declaration and its later amendments or comparable ethical standards.

Informed consent

The written informed consent was obtained from each patient or appropriate family member before the surgery.

\section{Funding}

The research had no sponsor support.

\section{References}

1. Ostrom QT, Patil N, Cioffi G, Waite K, Kruchko C, BarnholtzSloan JS. CBTRUS Statistical Report: Primary Brain and Other Central Nervous System Tumors Diagnosed in the United States in 2013-2017. Neuro Oncol. 2020 Oct 30;22(12 Suppl 2):iv1-iv96. doi: 10.1093/neuonc/noaa200

2. Bassiouni H, Hunold A, Asgari S, Stolke D. Meningiomas of the posterior petrous bone: functional outcome after microsurgery. J Neurosurg. 2004 Jun;100(6):1014-24. doi: 10.3171/jns.2004.100.6.1014

3. Agarwal V, Babu R, Grier J, Adogwa O, Back A, Friedman AH, Fukushima T, Adamson C. Cerebellopontine angle meningiomas: postoperative outcomes in a modern cohort. Neurosurg Focus. 2013 Dec;35(6):E10. doi: 10.3171/2013.10. FOCUS13367

4. Kane AJ, Sughrue ME, Rutkowski MJ, Berger MS, McDermott MW, Parsa AT. Clinical and surgical considerations for cerebellopontine angle meningiomas. J Clin Neurosci. 2011 Jun;18(6):755-9. doi: 10.1016/j.jocn.2010.09.023

5. He X, Liu W, Wang Y, Zhang J, Liang B, Huang JH. Surgical Management and Outcome Experience of 53 Cerebellopontine Angle Meningiomas. Cureus. 2017 Aug 3;9(8):e1538. doi: 10.7759 /cureus. 1538

6. D'Amico RS, Banu MA, Petridis P, Bercow AS, Malone H, Praver $M$, Wang TJC, Isaacson SR, Sisti MB. Efficacy and outcomes of facial nerve-sparing treatment approach to cerebellopontine angle meningiomas. J Neurosurg. 2017 Dec;127(6):12311241. doi: $10.3171 / 2016.10 . J N S 161982$

7. Hassaan SA, Tamura R, Morimoto Y, Kosugi K, Mahmoud 
M, Abokerasha A, Moussa A, Toda M, Yoshida K. Surgical outcomes of anterior cerebellopontine angle meningiomas using the anterior transpetrosal approach compared with the lateral suboccipital approach. Acta Neurochir (Wien). 2020 Jun;162(6):1243-1248. doi: 10.1007/s00701-020-04236-6

8. Sanai N, McDermott MW. A modified far-lateral approach for large or giant meningiomas of the posterior fossa. J Neurosurg. 2010 May;112(5):907-12. doi: 10.3171/2009.6.JNS09120

9. NakNakamura M, Roser F, Dormiani M, Matthies C, Vorkapic $P$, Samii M. Facial and cochlear nerve function after surgery of cerebellopontine angle meningiomas. Neurosurgery. 2005 Jul;57(1):77-90; discussion 77-90. doi: 10.1227/01. neu.0000154699.29796.34

10. Acioly MA, Liebsch M, Carvalho $\mathrm{CH}$, Gharabaghi A, Tatagiba M. Transcranial electrocortical stimulation to monitor the facial nerve motor function during cerebellopontine angle surgery. Neurosurgery. 2010 Jun;66(6 Suppl Operative):354-61; discussion 362. doi: 10.1227/01.neu.0000369654.41677.b7

11. Galloway GM, Nuwer MR, Lopez JR, Zamel KM. Intraoperative neurophysiologic monitoring. Cambridge University Press; 2010.

12. Schlake HP, Goldbrunner R, Siebert M, Behr R, Roosen K. Intra-Operative electromyographic monitoring of extraocular motor nerves (Nn. III, VI) in skull base surgery. Acta Neurochir (Wien). 2001;143(3):251-61. doi: 10.1007/ s007010170105

13. Li ZY, Li MC, Liang JT, Bao YH, Chen G, Guo HC, Ling F. Usefulness of intraoperative electromyographic monitoring of oculomotor and abducens nerves during skull base surgery. Acta Neurochir (Wien). 2017 Oct;159(10):1925-1937. doi: 10.1007/s00701-017-3268-z

14. Rampp S, Rensch L, Simmermacher S, Rahne T, Strauss C, Prell J. Intraoperative auditory steady-state monitoring during surgery in the cerebellopontine angle for estimation of postoperative hearing classes. J Neurosurg. 2017 Sep;127(3):559-568. doi: 10.3171/2016.7.JNS16460

15. Samii M, Gerganov VM. Cerebellopontine Angle Meningiomas. In: DeMonte F, McDermott MW, Al-Mefty O, editors. Al-Mefty's meningiomas. Thieme; 2011. doi: 10.1055/b-0034-81207 